\title{
A Design of Discrete-Time Adaptive Fuzzy Control System
}

\author{
Hugang HAN*1 Shuta MURAKAMI*2
}

\begin{abstract}
Recently various adaptive fuzzy control schemes have been proposed to deal with nonlinear systems with poorly understood dynamics by using the parameterized fuzzy approximator. However, all of the adaptive fuzzy control systems have been designed for a continue-time system, but actually it is realized by discrete-time control system where computers are the manipulators. So there has existed a gap between the design and realized control system. The goal of this paper is to design a discrete-time adaptive fuzzy control system. The adaptive laws to adjust parameters in the system will be developed based on Lyapunov synthesis approach. It is shown the proposed fuzzy adaptive controller guarantees tracking error, between outputs of considered system and desired values, to be asymptotically in decay.
\end{abstract}

Keywords: Fuzzy approximator, Adaptive control, Global stability, Nonlinear systems

\section{Introduction}

The application of fuzzy set theory to control problems has been the focus of numerous studies [1]. The motivation is often that the fuzzy set theory provides an alternative to the traditional modeling and design of control systems when system knowledge and dynamic models in the traditional sense are uncertain and time varying. Without any doubt, the stability is the most important requirement for any control system. It is highly concerned recently to design a fuzzy controller which guarantee the stability. Recently, some researches have been focused on use of the Lyapunov synthesis approach to construct stable adaptive fuzzy control system $[2,3,4,5,6]$. The most fundamental ideals of control algorithm are based on creative works[2,3]. Namely, to deal with the lack of control system, the fuzzy model would be considered as approximation model to approximating the unknown linear or nonlinear functions in the plant, and the fuzzy model is expressed as a serious of fuzzy basis function (FBF) expansion. Ultimately, instead of the unknown functions, the fuzzy models are utilized directly to construct the control inputs based on the Lyapunov synthesis approach.

However, the designed fuzzy control systems are actually realized by discrete-time control system because where the computers are the manipula-

* 1 School of Business, Hiroshima Prefectural University

*2 Faculty of Engineering, Kyushu Institute of Technology tors in practical applications, so there has existed a gap between the design and realized control system. Moreover, with the increasing applications of advanced computer technologies, it is much more meaningful to implement control system for nonlinear discrete-time systems. So far, however, as the literatures listed in the late this paper, all of the adaptive fuzzy control systems have been designed only for a continue-time system except using the so-called T-S type fuzzy model[7].

In this paper, we introduce a stable control algorithm in an effort to deal with discrete-time adaptive fuzzy control system. Herein, the control scheme considered here is the integration of fuzzy control component, in which the FBF expansion can be consider as an universal approximator, and sliding control component of the variable structure control with a sector $[8,9]$. The sector, defined by the state tracking errors of system, is reachable region of attraction around the switching hyperplane. Namely, the sector serves two purposes: one is to provide the global stability of the closedloop system when system goes the outside of the region where the global stability of system can not be guaranteed only by the force of fuzzy control, the other is to improve the tracking performance of the system within the FBF approximation region.

The remainder of this paper is arranged as follows. Section 2 describes a class of nonlinear uncertain discrete-time system to be controlled. Section 3 is a brief review of fuzzy system so that we can view the fuzzy system as an universal approximator. And we exploits the fuzzy system to 
develop adaptive control system in Section 4 in which we also give a detailed analysis of stability designed control system. Section 5 will show a computer simulation to verify the control algorithm given in Section 4, and conclusion is given in Section 6 in the paper.

\section{System Description}

We are interested in the single-input/singleoutput nonlinear discrete-time system

$$
\begin{gathered}
x(k+1)+f(x(k-n+1), x(k-n+2), \ldots, \\
x(k))=b u(k)
\end{gathered}
$$

where $k$ is current time, $x$ is the output, and $u$ is the input in the system. $f(\cdot)$ is nonlinear function with $n$ being system order. It is assumed that the order $n$ is known but the nonlinear function $f(\cdot)$ is unknown. It should be noted that more general classes of nonlinear systems could be transformed into this structure [10]. The control objective is to force the state vector $X(k)=\left[x_{1}(k), x_{2}(k), \ldots\right.$, $\left.x_{n}(k)\right]^{T}=[x(k-n+1), x(k-n+2), \ldots, x(k)]^{T}$ to follow a specified desired trajectory, $X_{d}(k)=$ $\left[x_{d}(k-n+1), x_{d}(k-n+2), \ldots, x_{d}(k)\right]^{T}$. Defining a traking error vector, $\tilde{X}(k)=X(k)-X_{d}(k)$, the problem is thus to design a control $u(k)$ which ensures that $\tilde{X}(k) \rightarrow 0$, as $k \rightarrow \infty$. For simplicity in this initial discussion, we take $b=1$ in the subsequent development.

Most of the results in adaptive control have focused on the situation where $f(X)$ lies in the span of a set of known (linear or nonlinear) regres. sors, or basis functions, $Y_{i}(X)$, i.e., $f(X)=\sum_{i=1}^{N}$ $\theta_{i} Y_{i}(X)$, where $\theta_{i}$ is a set of unknown parameters which appears linearly. Then, the function $f(X)$ can be approximated as $\hat{f}(X)=\sum_{i=1}^{N} \hat{\theta}_{i} Y_{i}(X)$, the approximation error only occurs on the parameters $\hat{\theta}_{i}$. Adaptive methods can then be used to adjust the parameters $\hat{\theta}_{i}$ to achieve the control objective. The challenge addressed in this paper is development of adaptive controllers when an explicit linear parameterization of the function $f(X)$ is either unknown or impossible. In the following section, it will be shown that usnig fuzzy IF-THEN rules, the unknown function $f(X)$ can be approximated by a parameterized fuzzy approximator. The parameters in this parameterized fuzzy approximator can then be stably tuned to provide effective tracking control architecture.

\section{Fuzzy Approximator}

We consider a fuzzy system for which there are four principal elements in such a fuzzy system : fuzzifier, fuzzy rule base, fuzzy inference engine, and defuzzifier. Let input space $X \in R^{n}$ be a compact product space. Assume that there are $N$ rules in the rule base and each of which has the following form :

$$
R_{j} \text { : IF } x_{1}(k) \text { is } A_{j}^{1} \text { and } x_{2}(k) \text { is } A_{j}^{2} \text { and }
$$
....... . and $x_{n}(k)$ is $A_{j}^{n}$, THEN $z(k)$ is $B_{j}$ where $j=1,2, \ldots, N, x_{i}(k)(i=1,2, \ldots, n)$ are the input variables to the fuzzy system at current time $k, z(k)$ is the output variable of the fuzzy system at current time $k$, and $A_{j}^{i}$ and $B_{j}$ are linguistic terms characterized by fuzzy membership functions $\mu_{\mathrm{A}^{i}(\mathrm{x})}$ and $\mu_{B_{i}}(z)$, respectively.

As in [11], we consider a subset of the fuzzy systems with singleton fuzzifier, product inference, and Gaussian membership function. Hence, such a fuzzy system can be written as

$$
h(X)=\frac{\sum_{j=1}^{N} \omega_{j}(k)\left(\prod_{i=1}^{n} \mu_{A_{j}}\left(x_{i}(k)\right)\right)}{\sum_{j=1}^{N} \prod_{i=1}^{n} \mu_{A_{j}}\left(x_{i}(k)\right)}
$$

where $h: U \subset R^{n} \rightarrow R, \omega_{j}(k)$ is the point in $R$ at which $\mu_{B_{j}}\left(\omega_{j}(k)\right)=1$, named as the connection weight $; \mu_{A_{j}}\left(x_{i}(k)\right)$ is the Gaussian membership function, defined by

$$
\mu_{A_{j}}\left(x_{i}(k)\right)=\exp \left[-\left(\frac{x(k)-\xi_{j}^{i}(k)}{\sigma_{j}^{i}(k)}\right)^{2}\right]
$$

where $\sigma_{j}^{i}(k)$ and $\boldsymbol{\xi}_{j}^{i}(k)$ are real-valued parameters, in which $\xi_{j}^{i}(k)$ indicates the position and $\sigma_{j}^{i}(k)$ indicates the variance of the membership

function. Here, if we take $\frac{\prod_{i=1}^{n} \mu_{A_{j}}\left(x_{i}(k)\right)}{\sum_{j=1}^{N} \prod_{i}^{n} \mu_{1}^{n} \mu_{A_{j}}\left(x_{i}(k)\right)}$ in

as basis functions and $\omega_{j}(k)$ as coefficients, $h(X)$, then, can be viewed as a linear combination of the basis functions. Therefore, we give a definition regarding the basis function as follows.

Definition 1 Define fuzzy basis functions $(F B F)$ as

$$
g_{j}(X)=\prod_{i=1}^{n} \mu_{A_{j}^{j}}\left(x_{i}(k)\right), j=1,2, \ldots, N
$$

where $\mu_{A_{i}}\left(x_{i}(k)\right)$ is the Gaussian membership functions defined in (3).

Then, the fuzzy system(2) is equivalent to a FBF 
expansion

$$
h(X)=\sum_{j=1}^{N} \omega_{j}(k) \cdot g_{j}(X) \triangleq W^{T}(k) \cdot G(X(k))
$$

where, $W(k)=\left[\omega_{1}(k), \omega_{2}(k), \cdots, \omega_{N}(k)\right]^{T}, G(X(k))$ $=\left[g_{1}(X(k)), \quad g_{2}(X(k)), \cdots, g_{N}(X(k))\right]^{T}$, and notation $\triangleq$ means a definition. For convenience, throughout this paper, the notation (.) is sometimes omitted when no confusion is likely to arise. For example we will express $G(X(k))$ as $G(k)$ with $k$ being current time.

We now show an important property of FBF expansion [3].

Theorem 1 For any given real continuous function $f$ on the compact set $U \in R^{n}$ and arbitrary $\varepsilon$, there exists optimal FBF expansion $h^{*}(X(k))=W^{*}(k)$. $G(X(k))$ such that

$$
\sup _{X \in U}\left|f(X(k))-h^{*}(X(k))\right|<\varepsilon
$$

This theorem states that the FBF expansion (5) is universal approximator on a compact set. Herein, we use terms fuzzy universal approximator or fuzzy approximator to refer to the FBF expansion. Since the fuzzy universal approximator is characterized by parameter vectors $W$, the optimal $h^{*}$ does contains an optimal vector $W^{*}$.

\section{Adaptive Fuzzy Control System}

\subsection{Structure of controller}

In this paper, we adopt the variable structure theory to construct our adaptive fuzzy control system. An error metric is firstly defined as

$$
s(k)=\left(q^{-1}+\lambda\right)^{n-1} \tilde{x}(k) \text { with } \lambda>0
$$

where $q^{-1}$ is the delay operator, and $\lambda$ defines the bandwidth of the error dynamics of the system. The error metric above can be rewritten as $s(k)=$ $\Lambda^{T} \tilde{X}(k)$ with $\Lambda^{T}=\left[\lambda^{n-1},(n-1) \lambda^{n-2}, \cdots, 1\right]$. The equation $s(k)=0$ defines a time-varying hyperplane in $R^{n}$ on which the tracking error vector $\widetilde{X}(k)$ decays exponentially to zero, so that perfect tracking can be asymptotically obtained by maintaining this condition[13]. In this case the control objective becomes the design of controller to force $s(k)=$ 0 .

An increment $\Delta s(k+1)$ can be written as

$$
\begin{aligned}
\Delta s(k+1) & =s(k+1)-s(k) \\
& =\Lambda_{1} \widetilde{X}(k)+\lambda^{n-1} \tilde{x}(k+1)
\end{aligned}
$$

where $\Lambda_{1}^{T}=\left[(n-1) \lambda^{n-2}-\lambda^{n-1}, \cdots, 1-(n-1) \lambda,-1\right]$. Substituting $\tilde{x}(k+1)=x(k+1)-x_{d}(x+1)$ into
(8) and combining (1) yield,

$$
\frac{1}{\lambda^{n-1}} \Delta s(k+1)=u(k)-h(k)
$$

where $h(k)$ denotes

$$
h(k)=f(X)+x_{d}(k+1)-\frac{1}{\lambda^{n-1}} \Lambda_{1}^{T} \tilde{X}(k)
$$

It naturally suggests that when $h(X)$ is known, a control input of form

$$
u(k)=-k_{d} s(k)+h(k), k_{d}>0
$$

leads to a closed-loop system $\Delta s(k)=-k_{d} s(k)$, and hence, $\widetilde{X}(k) \rightarrow 0$ as $k \rightarrow \infty$. The problem is how $u(k)$ can be determined when $h(X)$, which concludes an unknown functions $f(X)$ and $b$, are unknown. Therefore we have to approximate them to achieve control objective. Here the fuzzy approximator described in the previous section is used. Let us denote $h^{*}(X(k))=W_{h}^{* T} G_{h}(k)$ to be the optimal fuzzy approximator of the unknown function $h(X(k))$. However, we have no idea to know the optimal parameter vector $W_{h}^{*}$ in the optimal fuzzy approximator. Generally, the estimate, denoted $\hat{h}(X(k))=\hat{W}_{h}^{T} G_{h}(k)$, is adopted instead of the optimal fuzzy approximator $h^{*}(X(k))$. Regarding the optimal fuzzy approximator, we make following assumptions.

Assumption 1 The optimal fuzzy approximator error, $d_{h}(k)=h^{*}(X(k))-h(X(k))$, satisfy the following inequality, where $\varepsilon_{h}^{*}$ is known small positive value.

$$
\sup _{X \in \ell}\left|d_{h}(k)\right|<\varepsilon_{h}^{*}
$$

Assumption 2 In defined subspace $\mathcal{C}_{w_{h}} \ni W_{h}^{*}, \quad \mathcal{C}_{\varepsilon_{h}} \ni$ $\varepsilon_{h}^{*}$, there are some positive constants $c_{w_{n}}, c_{\varepsilon_{n}}$, that satisfies the following inequalities.

$$
\begin{aligned}
& \left\|\hat{W}_{h}(k)-W_{h}^{*}\right\|_{2} \leq c_{w_{h}}, \quad \forall \hat{W}_{h}(k) \in \mathcal{C}_{w_{h}} \\
& \left|\hat{\varepsilon}_{h}(k)\right|+\left|\varepsilon_{h}^{*}\right| \leq c_{\varepsilon_{h}}, \quad \forall \hat{\varepsilon}_{h}(k) \in \mathcal{C}_{\varepsilon_{h}}
\end{aligned}
$$

where $\hat{\varepsilon}_{h}(k)$ is the estimate of $\varepsilon_{f}^{*}$, as well as $\hat{W}_{h}(k)$.

Remarks:

1. Base the Theorem 1 , the assumption 1 is reasonable to provide.

2. It seems the assumption 2 is difficult to satisfy. However, in the each defined subspace where the optimal parameter (or vector) is contained, such an inequality is easy to check. Therefore, the problem is how to force the each estimate to enter the each defined subspace. At the upcoming algorithm, the projection algorithm is adopted to ensure each estimate is within the 
each defined subspace.

Inspired by the above control structure in (11), using the fuzzy approximator $\hat{h}(X)$, our adaptive control law is now described below:

$$
u(k)=u_{f u}(k)+u_{v u}(k)
$$

where $u_{f u}(k)$, expressed by ,

$$
\begin{aligned}
u_{f u}(k) & =\hat{h}(k)-\hat{\varepsilon}_{h}(k) \operatorname{sgn}(s(k)) \\
& =\hat{W}_{h}^{T}(k) G_{h}(k)-\hat{\varepsilon}_{h}(k) \operatorname{sgn}(s(k))
\end{aligned}
$$

is fuzzy component of control law which will attempt to recover or cancel the unknown function $h$. And the fuzzy component is synthesized by

$$
\begin{aligned}
& \hat{W}_{h}(k)=\wp\left\{\hat{W}_{h}(k-1)-\Gamma_{h} G_{h}(k) s(k)\right\} \\
& \hat{\varepsilon}_{h}(k)=\wp\left\{\hat{\varepsilon}_{h}(k-1)+\gamma_{h}|s(k)|\right\}
\end{aligned}
$$

where $\hat{W}_{h}$, and $\hat{\varepsilon}_{h}$ are the estimates of $W_{h}^{*}$, and $\varepsilon_{h}^{*}$, respectively $; \wp$ represents the projection operator necessary to ensure that $\hat{W}_{h}(k) \in \mathcal{C}_{w_{n}}$, and $\hat{\varepsilon}_{h}(k) \in \mathcal{C}_{\varepsilon_{h}}$ for $\forall k[14] ; \Gamma_{h} \in R^{N \times N}$, and $\gamma_{h}>0$ determine the rates of adaptation in which $\Gamma_{h}$ is a symmetric positive define matrix and selected to be satisfy the following inequality :

$$
G_{h}^{T}(k) \Gamma_{h} G_{h}(k) \leq \frac{2}{\lambda^{n-1}}
$$

It should note that the term like $\hat{\varepsilon}_{n}(k)$ $\operatorname{sgn}(s(k))$ in (16) actually reflects the component for compensation of the approximating error $(\hat{h}$ $(k)-h(k))$. If the fuzzy approximator provide good description of the unknown function $h(k)$, then the term $\hat{\varepsilon}_{h}(k)$ should be small as well as the optimal fuzzy approximator error $d_{h}(k)$. Conversely, if the fuzzy approximator is poor, $\hat{\varepsilon}_{h}(k)$ will rise automatically to the necessary level, ensuring the stability of the overall system.

$u_{v u}(k)$ is the variable structure control component that is expressed by

$$
u_{v u}(k)=\beta_{h 1} c_{w_{h}}\left\|G_{h}(k)\right\|+\beta_{h 2} c_{\varepsilon_{h}}
$$

The variable structure control component only works outside of the sector $\Omega(k)[8]$ defined by

$$
\Omega(k)=\Omega_{h 1}(k) \cup \Omega_{h 2}(k)
$$

with

$$
\begin{aligned}
& \Omega_{h 1}(k)=\left\{s(k)||\left\|G_{h}(k)\right\| s(k) \mid c_{w_{h}} \leq \rho_{h 1}\right\} \\
& \Omega_{h 2}(k)=\left\{s(k)|| s(k) \mid c_{\varepsilon_{h}} \leq \rho_{h 2}\right\} \\
& \rho_{h 1}=\frac{\lambda^{n-1}(\ell+1)^{2} c_{w_{h}}}{2 \ell}\left\|G_{h}(k)\right\| A(k) \\
& \rho_{h 2}=\frac{\lambda^{n-1}(\ell+1)^{2} c_{\varepsilon_{h}}}{2 \ell} A(k) \\
& A(k)=c_{w_{h}}\left\|G_{h}(k)\right\|+c_{\varepsilon_{h}}
\end{aligned}
$$

where $\ell$ is a positive constant chosen to guarantee the system robustness. And the switching type coefficients in (20) are determined as follows.

$$
\begin{aligned}
& \beta_{h 1}=\left\{\begin{array}{r}
\ell, \text { for } c_{w_{h}}\left\|G_{h}(k)\right\| s(k)<-\rho_{h 1} \\
0, \text { for } c_{w_{h}}\left|\left\|G_{h}(k)\right\| s(k)\right| \leq \rho_{h 1} \\
-\ell, \text { for } c_{w_{h}}\left\|G_{h}(k)\right\| s(k)>\rho_{h 1}
\end{array}\right. \\
& \beta_{h 2}=\left\{\begin{aligned}
\ell, & \text { for } c_{\varepsilon_{h}} s(k)<-\rho_{h 2} \\
0, & \text { for } c_{\varepsilon_{h}}|s(k)| \leq \rho_{h 2} \\
-\ell, & \text { for } c_{\varepsilon_{h}} s(k)>\rho_{h 2}
\end{aligned}\right.
\end{aligned}
$$

Remarks :

1. In contrast with the continuous-time adaptive fuzzy control system[6], the term of variable structure control component $u_{v u}(k)$ corresponds to the sliding component $u_{s u}(t)$. In [6], via a modulate $m(t)$ the $u_{s u}(t)$ only works on the specified region $A_{d}^{c}$ where the fuzzy approximator could not effectively approximate the unknown function in a sense. Similarly, here $u_{v u}(k)$ only works outside of the sector $\Omega(k)$ which is defined on the tracking error metric $s(k)$ via the switching type coefficients $(27 \sim 28)$.

2. Compared with the other adaptive schemes given in [8] [9], there is an important difference. In $[8][9]$, the convergence of tracking error metric depends on a assumption like $\sup _{j} \widetilde{\boldsymbol{\omega}}_{j}(k) \leq$ $d$, where $d$ is a known constant, which may not be easy to check, because it could not ensure that $\hat{\omega}_{j}$ is bounded. On the other hand, in our scheme, by using a projection algorithm we can easily realize $\hat{W}(k) \in \mathcal{C}_{w}$ so that $\|\widetilde{W}(k)\|$ $<c_{\omega}$. This will enhance the flexibility of the system.

\subsection{Analysis of stability}

The stability of the closed-loop system described by (1) and (15-28) is established in the following theorem.

Theorem 2 Under the assumptions 1 and 2, if the plant (1) is controlled by (15), (16), (20), (2728), and the adaptive component is synthesized by (17-18), then the tracking error metric of the system will stably enter the sector defined by (21-26). When the system tracking error metric is driven inside the sector, $|s(k+1)|$ is usually in a small magnitude such that it can be assumed that $|\Delta s(k+1)| \leq\left(\left(\gamma_{h}-k_{d}\right) \lambda^{n-1}\right)^{1 / 2}|s(k)|$ with $0<$ $k_{d}<\gamma_{n}$. Then, $\tilde{X}(k) \rightarrow 0$ as $k \rightarrow \infty$ and the all signals are bounded.

\section{Proof :}

From (9), (15), and (16), we have

$$
\frac{1}{\lambda^{n-1}} \Delta s(k+1)
$$




$$
\begin{aligned}
= & -\widetilde{W}_{h}^{T}(k) G_{h}(k)-\hat{\varepsilon}_{h}(k) \operatorname{sgn}(s(k)) \\
& +d_{h}(k)+u_{v u}(k)
\end{aligned}
$$

where $\widetilde{W}_{h}(k)=W_{h}^{*}-\hat{W}_{h}(k)$.

Now, consider the following Lyapunov function candidate,

$$
\begin{aligned}
V(k)= & \frac{1}{2}\left(\frac{1}{\lambda^{n-1}} s^{2}(k)\right. \\
& +\widetilde{W}_{h}^{T}(k-1) \Gamma_{h}^{-1} \widetilde{W}_{h}(k-1) \\
& \left.+\gamma_{h}^{-1} \widetilde{\varepsilon}_{h}^{2}(k-1)\right)
\end{aligned}
$$

where $\widetilde{\varepsilon}_{h}(k)=\varepsilon_{h}^{*}-\hat{\varepsilon}_{h}(k)$. Then the increment $\Delta V(k+1)=V(k+1)-V(k)$ becomes

$$
\begin{aligned}
\Delta V & (k+1) \\
= & \underbrace{\frac{1}{2 \lambda^{n-1}}\left(s^{2}(k+1)-s^{2}(k)\right)}_{(1)} \\
& +\underbrace{\frac{1}{2}\left(\widetilde{W}_{h}^{T}(k) \Gamma_{h}^{-1} \widetilde{W}_{h}(k)-\widetilde{W} W_{h}^{T}(k-1) \Gamma_{h}^{-1} \widetilde{W}_{h}(k-1)\right)}_{(11)} \\
& +\underbrace{\frac{1}{2} \gamma_{h}^{-1}\left(\widetilde{\varepsilon}_{h}^{2}(k)-\widetilde{\varepsilon}_{h}^{2}(k-1)\right)}_{\text {(III) }}
\end{aligned}
$$

Further,

$$
\begin{aligned}
(I)= & \frac{1}{\lambda^{n-1}} \Delta s(k+1) s(k) \\
& +\frac{1}{2 \lambda^{n-1}} \Delta^{2} s(k+1) \\
& +\frac{1}{2 \lambda^{n-1}} s^{2}(k)
\end{aligned}
$$

Multiplying both sides of (29) by $s(k)$ and substituting it into (32), it follows

$$
\begin{aligned}
(I)= & u_{v u}(k) s(k)+\frac{1}{2 \lambda^{n-1}} \Delta^{2} s(k+1) \\
& +\frac{1}{2 \lambda^{n-1}} s^{2}(k)-\widetilde{W}_{h}^{T}(k) G_{h}(k) s(k) \\
& -\hat{\varepsilon}_{h}(k)|s(k)|+d_{h}(k) s(k)
\end{aligned}
$$

Here, let $\hat{W}_{n p}(k)$ denote the parameter estimate vector before projection, i.e., $\hat{W}_{h}(k)=\wp\left\{\hat{W}_{n p}(k)\right\}$. Thus,

$$
\begin{aligned}
& \hat{W}_{n p}(k)-\hat{W}_{h}(k-1) \\
& =-s(k) \Gamma_{f} G_{h}(k) \triangleq \Delta \hat{W}_{h}(k)
\end{aligned}
$$

and considering the relations like $\hat{\varepsilon}_{n p}(k)-$ $\hat{\varepsilon}_{h}(k-1)=\gamma_{h}|s(k)|$, we have

$$
\begin{aligned}
(\mathrm{II}) \leq & \frac{1}{2}\left(\widetilde{W}_{h}^{T}(k) \Gamma_{h}^{-1} \widetilde{W}_{n p}(k)\right. \\
& \left.-\widetilde{W}_{h}^{T}(k-1) \Gamma_{h}^{-1} \widetilde{W}_{h}(k-1)\right) \\
\leq & \frac{1}{2}\left(\widetilde{W}_{h}^{T}(k) \Gamma_{h}^{-1} \widetilde{W}_{n p}(k)\right. \\
& +\widetilde{W}_{n p}^{T}(k) \Gamma_{h}^{-1} \widetilde{W}_{h}(k-1) \\
- & \widetilde{W}_{h}^{T}(k) \Gamma_{h}^{-1} \widetilde{W}_{h}(k-1) \\
& \left.-\widetilde{W}_{h}^{T}(k-1) \Gamma_{h}^{-1} \widetilde{W}_{h}(k-1)\right)
\end{aligned}
$$

$$
\begin{aligned}
= & \frac{1}{2}\left(-\widetilde{W}_{h}^{T}(k) \Gamma_{h}^{-1} \Delta \hat{W}_{h}(k)\right. \\
& \left.-\Delta \hat{W}_{h}^{T}(k) \Gamma_{h}^{-1} \widetilde{W}_{h}(k-1)\right) \\
= & -\Delta \hat{W}_{h}^{T}(k) \Gamma_{h}^{-1} \widetilde{W}_{h}(k) \\
& -\frac{1}{2} \Delta \hat{W}_{h}^{T}(k) \Gamma_{h}^{-1} \Delta \hat{W}_{h}(k) \\
= & s(k) G_{h}^{T}(k) \widetilde{W}_{h}(k) \\
& -\frac{1}{2} G_{h}^{T}(k) \Gamma_{h} G_{h}(k) s^{2}(k)
\end{aligned}
$$

where $\Delta \widetilde{W}_{h}(k)=-\Delta \hat{W}_{h}(k)$ has been used. Similarly, by the same way we can obtain the inequality as follows.

$$
(\mathrm{III}) \leq-|s(k)|\left(\varepsilon_{h}^{*}-\hat{\varepsilon}_{h}(k)\right)-\frac{1}{2} \gamma_{h} s^{2}(k)
$$

Therefore, $\Delta V(k+1)$ becomes that,

$$
\begin{aligned}
\Delta V & (k+1) \leq-\frac{1}{2} \gamma_{h} s^{2}(k) \\
& -\frac{1}{2} G_{h}^{T}(k) \Gamma_{h} G_{h}(k)+\frac{1}{2 \lambda^{n-1}} s^{2}(k) \\
& +\frac{1}{2 \lambda^{n-1}} \Delta^{2} s(k+1)+u_{v u}(k) s(k) \\
& -|s(k)| \varepsilon_{h}^{*}+d_{h}(k) s(k) \\
\leq & -\frac{1}{2} \gamma_{h} s^{2}(k) \\
& +\frac{1}{2 \lambda^{n-1}} \Delta^{2} s(k+1)+u_{v u}(k) s(k)
\end{aligned}
$$

where the facts that inequality (19), and $\left|d_{h}(k)\right|$ $<\varepsilon_{h}^{*}$ have been used.

If $\Delta V(k+1)$ satisfies the relations like $\Delta V(k+1)$ $\leq-\frac{1}{2} \gamma_{n} s^{2}(k)$, using the Barbalat's lemma [15] we can easily show that $s(k)$ will be decreased, and $s(k) \rightarrow 0$ as $k \rightarrow \infty$. It also means that

$$
\lim _{k \rightarrow \infty} \tilde{X}(k)=0
$$

To complete this proof, we now consider two cases: (a) the outside of the sector $\Omega$, (b) the inside of the sector $\Omega$, and show the stability of the system and the convergence of tracking error.

(a) In case of the outside of the sector $\Omega$ : From (20) and (27-28), we have

$$
\begin{aligned}
u_{v u}(k)_{s}(k) & \leq-\left|\beta_{h 1} \rho_{h 1}\right|-\left|\beta_{h 2} \rho_{h 2}\right| \\
& =-\ell \rho_{h 1}-\ell \rho_{h 2} \\
& =-\frac{\lambda^{n-1}(\ell+1)^{2}}{2} A^{2}(k)
\end{aligned}
$$

On the other hand, from (20) and (29), we get

$$
\begin{aligned}
& \left|\lambda^{n-1} \Delta s(k+1)\right| \\
& \quad \leq\left|u_{v u}(k)\right|+c_{w_{h}}\left\|G_{h}(k)\right\|+\left|\varepsilon_{h}^{*}+\hat{\varepsilon}_{h}(k)\right| \\
& \quad \leq\left|u_{v u}(k)\right|+c_{w_{h}}\left\|G_{h}(k)\right\|+c_{\varepsilon_{h}} \\
& \quad=(\ell+1) A(k)
\end{aligned}
$$

where $\left|d_{h}(k)+\hat{\varepsilon}_{h}(k) \operatorname{sgn}(s(k))\right| \leq \mid \varepsilon_{h}^{*}+\hat{\varepsilon}_{h}(k) \operatorname{sgn}$ 
$(s(k)) \mid \leq c_{\varepsilon_{h}}$, which depends on the assumption 2 , has been used.

Therefore,

$$
\frac{1}{2 \lambda^{n-1}} \Delta^{2} s(k+1) \leq \frac{\lambda^{n-1}}{2}(\ell+1)^{2} A(k)
$$

Combining (42), (39) and (37) yields

$$
\Delta V(k+1) \leq-\frac{1}{2} \gamma_{h} s^{2}(k)
$$

It means that the error state will decrease and enter the inside of the sector.

(b) In case of the inside of the sector $\Omega$ :

When the system tracking error metric is driven inside the sector, $|s(k+1)|$ is usually in a small magnitude such that it can be assumed that $|\Delta s(k+1)| \leq\left(\left(\gamma_{h}-k_{d}\right) \lambda^{n-1}\right)^{1 / 2}|s(k)|$ with $0<k_{d}<$ $\gamma_{h}$. Then from (37), we get

$$
\Delta V(k+1) \leq-\frac{1}{2} k_{d} s^{2}(k)
$$

with $u_{v u}(k)=0$. Clearly, (38) can be obtained. In addition, from $(30),(43)$ and (44), we can conclude that the all parameters are bounded.

\section{Simulation Example}

To illustrate and clarify the proposed design procedure, we apply the adaptive fuzzy controller developed in the Section 4 to a nonlinear system [3]:

$$
x(k+1)=\frac{1-e^{-x(k)}}{1+e^{-x(k)}}+u(k)
$$

where $f=\frac{1-e^{-x(k)}}{1+e^{-x(k)}}$ is a nonlinear function. The control objective is to force the system state to follow the reference input $x_{d}(k)=\sin (k)$. The simulation is conducted with the following linguistic description :

$R_{j}$ :IF $x$ is near $k$ THEN $f$ is near $B_{k}$ where near $k=-2,-1,0,1,2$ are five fuzzy sets with membership functions $\mu_{k}(x)=\exp \left(-\left(\frac{x-k}{\sigma}\right)^{2}\right)$ as shown in Fig.1. To verify the ability of our adaptive fuzzy controller, we consciously give a set of unsuitable values $W_{h}(0)=[-4,-2,0,4,6]^{T}$ for consequences of fuzzy rules. As the behavior to approximate the nonlinear function $f$ with the initial value $W_{h}(0)$, apparently, the fuzzy system $\hat{f}$ $\left(=\hat{h}-x_{d}(k+1)+\frac{1}{\lambda^{n-1}} \Lambda_{1}^{T} \widetilde{X}(k)\right)$ can not approximate $f$ effectively(Fig.4). In this simulation, we define a sphere whose radius is 1 as subspace $C_{w_{n}}$ where $W_{h}^{*}$ and $\hat{W}_{h}(k)$ are within so that $c_{w_{n}}=\sqrt{2}$ by
(13). By the same token, we suppose that $\varepsilon_{h}^{*}$ and $\hat{\varepsilon}_{h}(k)$ are within a defined subspace $\mathcal{C}_{\varepsilon_{h}}$ whose radius of the sphere is 0.1 so that $c_{\varepsilon_{h}}=0.2$ by (14). In addition, we take the values that $\ell=1$ and initial state $x 0=2$.

The results of simulation are shown in Fig.2-3. Fig. 2 are the evolution of $x(k)$ and the reference input

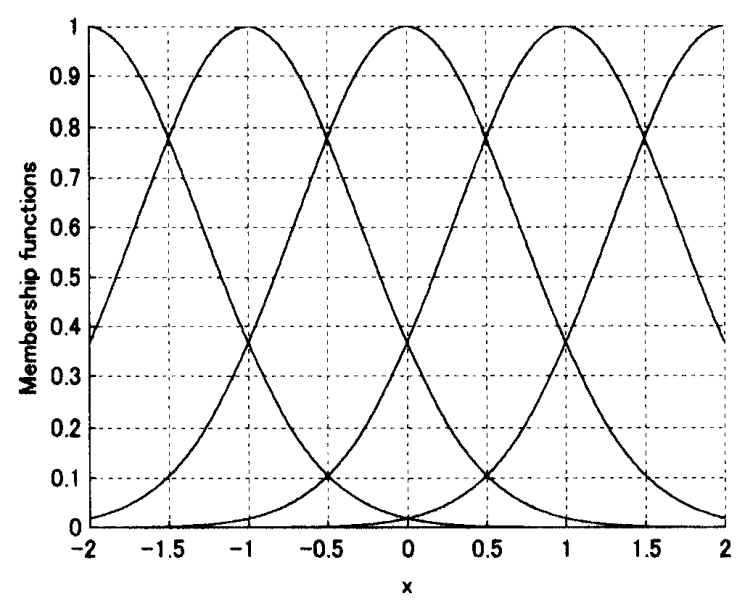

Figure 1: Membership functions

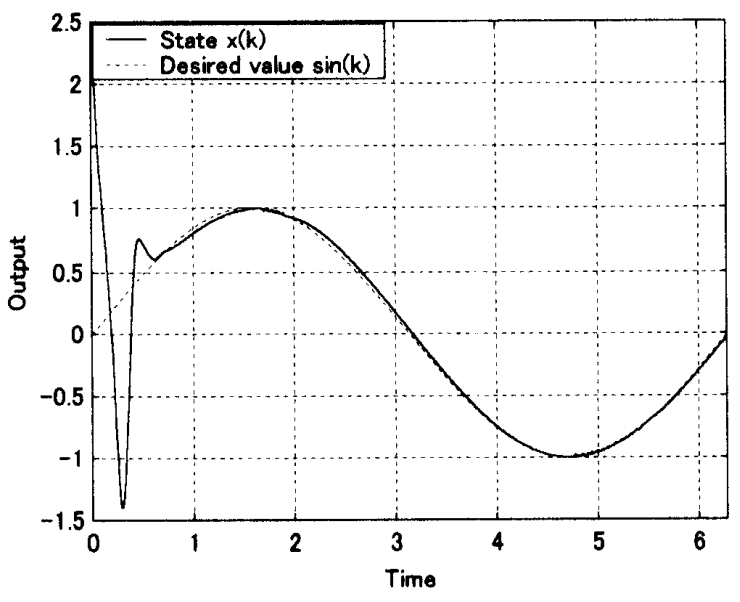

Figure 2: Closed-loop state $x(k)$

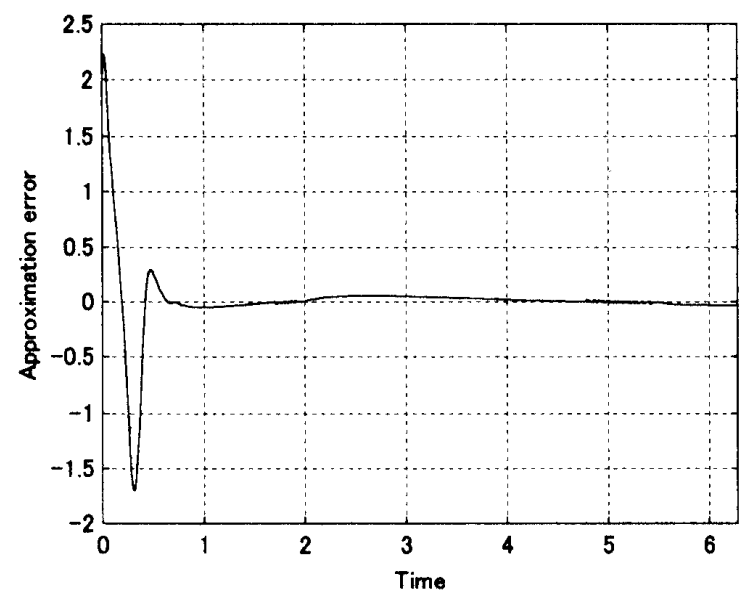

Figure 3 : Error between $f(k)$ and $\hat{f}(k)$ 
$x_{d}(k)$ of the system, and Fig. 3 shows the transit of the approximating error $(f(x)-\hat{f}(x))$. It can been seen from the simulation results that the nonlinear is successfully controlled using the adaptive fuzzy controller proposed in this paper, even based on the unsuitable the initial fuzzy system.

In addition, the aspect of fuzzy system with the final tuned consequences is shown in Fig.4. Comparing with the initial fuzzy system (dotted line), the performance to approximate $f(k)$ is improved drastically.

\section{Conclusion}

In this paper, we proposed a design for a class of discrete-time adaptive fuzzy control system and the parameters in FBF expansion could be stably tuned. The developed controller guarantees the global stability of the resulting closed-loop system in the sense that all signals involved are uniformly bounded and tracking error would be asymptotically in decay. Hereafter, we will extend our algorith$m$ developed in this paper to fuzzy predictive control systems.

\section{References}

[1]I. J. Leontaritis and S. A. Billings, "Input-output parametric models for nonlinear systems. Part I and II," International Joumal of Control, vol.41, pp. 303-344, 1985.

[2]L.X. Wang, "Stable adaptive fuzzy control of nonlinear system," IEEE Trans. on Fuzzy Systems, vol.1, pp.146-155, 1993.

[ 3 ] C.-Y. Su and Y. Stepanenko, "Adaptive control of a class of nonlinear systems with fuzzy logic, "IEEE Trans. on Fuzzy Systems, vol.2, pp.258-294, 1994.

[4]Y.-G. Piao, H.-G. Zhang and Z. Bien, "Design of fuzzy direct controller and stability analysis for a class of nonlinear system," Proceedings of the ACC, pp.2274-2275, USA, 1998.

[5]T. Chai and S. Tong, "Fuzzy direct adaptive control for a class of nonlinear systems," Fuzzy Sets and Systems, vol.103, no.3, pp.379-384, 1999.

[6]H. Han, C.-Y. Su and S. Murakami, "Further results on adaptive control of a class of nonlinear systems with fuzzy logic," Journal of Japan Society for Fuzzy Theory and System, vol.11, no.5, pp.501 $-509,1999$.

[ 7 ] S.-J. Wu and C.-T. Lin, "Global stability analysis of discrete time-invariant optimal fuzzy control systems," International Journal of Fuzzy Systems, vol. 1 , no.1, pp.60-68, 1999.\}

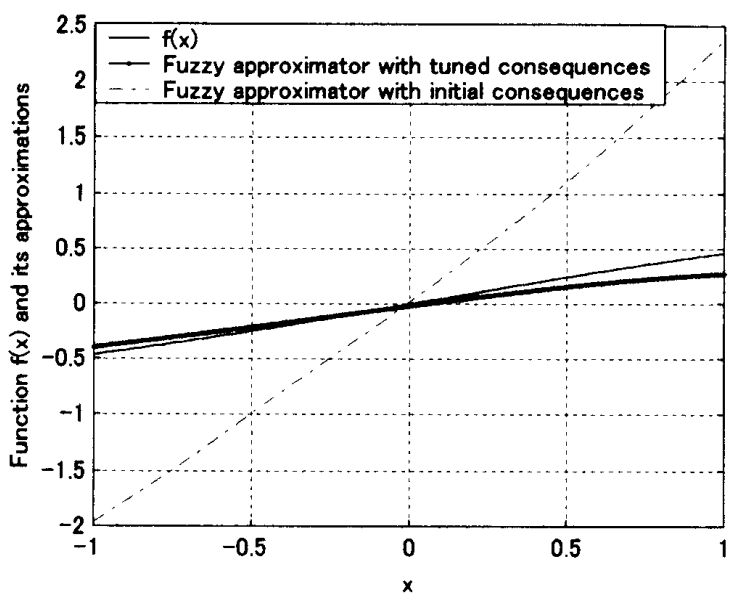

Figure 4 : Behaviors of fuzzy approximators

[8] Katsuhisa Furuta, "VSS type self-tuning control," IEEE Trans. on Industrial Electronics, vol.40, no. 1, pp.37-44, 1993.

[9]F. Sun, Z. Sun, and P.-Y. Woo, "Stable neuralnetwork-based adaptive control for sampled-data nonlinear systems," IEEE Trans. on Neural Networks, vol.9, no.5, pp.956-968, 1998.

[10]S. Sastry and M. Bodson, Adaptive Control, Prentice Hall, 1989.

[11] L.-X. Wang and J. M. Mendel, "Back-propagation fuzzy system as nonlinear dynamic system identifiers," Proc. of IEEE Conf. on Fuzzy Systems, pp.1409-1418, San Diego, 1992.

[12] C.-J. Chien and L.C. Fu, "Adaptive control of interconnected systems using neural network design,"

Proc. of 37th IEEE Conf. on Decision and Control, Tampa, TL. 1998.

[13] J.J. Slotine and J. A. Coetsee, "Adaptive sliding controller synthesis for nonlinear systems," Int. J Control, vol.43, pp.1631-1651, 1986.

[14] Changyun Wen, "A robust adaptive controller with minimal modification for discrete time-varying systems," IEEE Trans. on Auto. Control, vol.39, no. 5, pp.987-991, 1994.

[15]J.-J.E.Slotine and W. Li, Applied nonlinear control, Prentice Hall, 1991.

(March 1, 2000 received)

[Contact Address]

Hugang HAN

School of Business,

Hiroshima Prefectural University

562 Nanatsuka, Shobara-shi, Hiroshima 727-0023, Japan

TEL : 08247-4-1706

FAX : 08247-4-0191

E-mail : kan@bus.hiroshima-pu.ac.jp 
Information about Authors

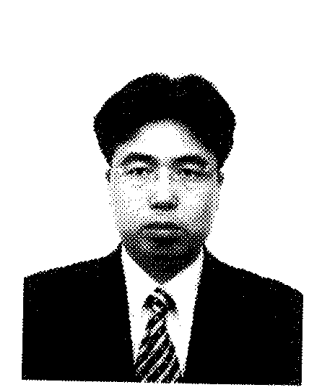

Hugang HAN [Member]

Hugang Han received the M.S. degree in mechanical engineering from Yamagata University, in 1992 , and Ph.D. degree in electric engineering form Kyushu Institute of Technology, in 1997. From 1992 to 1994 he was with the Department of Automation at Northeast China Institute of Electric Power Engineering. Since 1997, he has been at Hiroshima Prefectural University where he is currently an Associate Professor of the Department of Management and Information Science. Dr. Han's main research interests are in fuzzy control, adaptive control and neurofuzzy system.

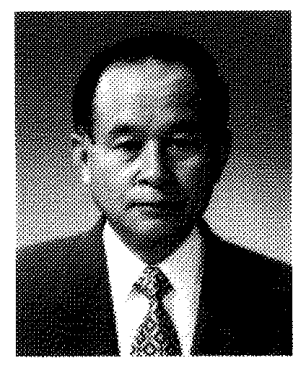

Shuta MURAKAMI [Member]

Shuta Murakami received Ph.D. degree in control engineering from Tokyo Institute of Technology, in 1969. Since 1969, he has been with the Kyushu Institute of Technology, where he was a Lecturer with the Department of Control Engineering from 1969 to 1970 , an Associate Professor from 1970 to 1984 . Since 1984, he has been a Professor with the Department of Computer Science. Dr. Murakami's main research interests are in fuzzy control, fuzzy modeling, fuzzy decision-making and so on. 